\title{
Gouvernance de l'eau et maladies hydriques dans les campagnes des Hautes Terres de l'Ouest Cameroun:Analyse à partir du cas du village Bangoua
}

\author{
Feukem Tchinda Agnès, \\ Yemmafouo Aristide,
}

Centre d'Etudes et de Recherche en Espace,

Arts et Humanités (CEREAH), Université de Dschang, Cameroun

Doi:10.19044/esj.2020.v16n17p182 URL:http://dx.doi.org/10.19044/esj.2020.v16n17p182

\section{Résumé}

L'absence du système d'approvisionnement en eau potable dans certains milieux ruraux africains y justifie le taux élevé des maladies hydriques africains. L'objectif de cette étude est d'établir la corrélation entre l'évolution de la gouvernance de l'eau et celle des maladies hydriques dans les phases du projet Scanwater, du projet de rehabilitation du reseau scanwater dans les Hautes Terres de l'Ouest-Cameroun. Pour atteindre cet objectif, 153 ménages ont été enquêtés suivant un quota de 25 par quartiers. Les observations ont permis de déterminer le comportement des populations sur l'utilisation de l'eau. La sélection des quartiers enquêtés et les données sur les maladies hydriques ont été obtenues grâce aux registres du comité de gestion et du centre de santé. Il est démontré que le projet de réhabilitation a contribué à une baisse significative des maladies hydriques tandis que l'arrêt de fonctionnement a favorisé leur croissance. Lorsque des comités de gestion participative se mettent sur pieds et fonctionnement normalement, les maladies hydriques chutent. Le centre de santé locale a enregistré 985 cas de maladies hydriques entre 2002-2004, contre seulement 314 entre 2005-2007 lorsque ces comités de gestion participative ont été mis sur pieds. Cependant, à partir de 2008, les crises de gestion reprennent et le nombre de malade progresse pour atteindre 1154 en 2018. L'index d'efficacité de la gouvernance de l'eau et le rapport aux maladies hydriques est de 41 en 2007 contre 9 en 2018 montrant donc que la gouvernance de l'eau a une corrélation significative avec l'augmentation ou la diminution des maladies hydriques.

Mots-clés : Gouvernance de l'eau, eau potable, maladies hydriques, OuestCameroun 


\title{
Water Governance and Waterborne Diseases in the Rural Areas of the Western Highlands of Cameroon: Analysis Based on the Case of Bangoua Village
}

\author{
Feukem Tchinda Agnès, \\ Yemmafouo Aristide,
}

Centre d'Etudes et de Recherche en Espace, Arts et Humanités (CEREAH), Université de Dschang, Cameroun

\begin{abstract}
The lack of safe drinking water supply justifies the high rate of waterborne diseases in rural Africa. The objective of this research is to establish the correlation between the evolution of water governance and that of waterborne diseases in the phases of the Scanwater project and its rehabilitation in the West Cameroon Highlands. To achieve this objective, 153 households were surveyed with a quota of 25 per neighbourhood. The observations made it possible to determine the behaviour of the population with regard to water use. The selection of the surveyed neighbourhoods and data on waterborne diseases were obtained from the registers of the management committee and the health centre. It is demonstrated that the rehabilitation project contributed to a significant decrease in waterborne diseases, while the shutdown of operations has favoured their exponential growth. When participatory management committees are set up and functioning normally, waterborne diseases drop. The local health centre recorded 985 cases of waterborne diseases between 2002-2004, compared to only 314 between 2005-2007 when these participatory management committees were set up. However, from 2008 onwards, management crises resume and the number of patients increases to reach 1154 in 2018. The water governance effectiveness index and the ratio of waterborne diseases is 41 in 2007 against 9 in 2018, thus showing that water governance has a significant correlation with the increase or decrease in waterborne diseases.
\end{abstract}

Keywords: Water governance, drinking water, waterborne diseases, West Cameroon

\section{Introduction}

Dans les pays d'Afrique subsaharienne $70 \%$ des maladies sont d'origine hydrique (OMS, 2010) et plus de 1,8 millions de personnes en 
meurent chaque année, ce qui en fait la première cause de mortalité (OMS, 2006). Toujours dans cette région, le taux d'accès à l'eau potable et à l'assainissement est le plus faible du monde (OMS, 2006), ce qui en fait la partie du monde la plus désavantagée en matière d'approvisionnement en eau (Yongsi, 2010). Cependant, il s'agit d'un des plus grands bassins d'eau au regard du réseau hydrographique dense et du montant annuel des précipitations. Le problème n'est donc pas tant la disponibilité, mais l'accessibilité à l'eau (Van der Bliek et al. 2007, Catherine, 2011, Nkengfack, 2017). Lorsque sa bonne gestion n'est pas assurée, elle peut véhiculer un très grand nombre d'agents pathogènes à l'origine de maladies hydriques (Goita, 2014 ; Vissin et al., 2016).

Le principe de bonne gouvernance de l'eau repose, selon le document 7 du Global Water Partnership dans son introduction (2003), sur 06 principes fondamentaux parmi lesquelles: l'amélioration de la participation des acteurs aux décisions, la cohérence et synergie des politiques et dispositions juridiques, l'équité et l'éthique, la clarification des processus menant aux décisions, la recherche de la durabilité des actions, la transparence, la pertinence et la qualité des institutions. La Gestion Intégrée des Ressources en Eaux est le cadre d'opérationnalisation de ces principes. Elle vise à améliorer l'efficience dans l'utilisation de l'eau (raison économique), promouvoir l'équité dans l'accès à l'eau (raison sociale) et garantir la durabilité (raison environnementale). Elle veille en priorité au respect des intérêts des «ayants droits » (citoyens, pouvoirs publics, partenaires...

Le Cameroun dispose environ 322 milliards de $\mathrm{m}^{3}$ des ressources en eau et les ressources souterraines constituent 21\% (Mafany et Fantong, 2006), soit un ratio de $21000 \mathrm{~m}^{3} \mathrm{~d}$ 'eau par habitant par an. Ce ratio contraste avec le déficit d'approvisionnement. Le taux d'accès à l'eau potable atteint à peine 77 $\%$ de la population. Le milieu rural est le plus désavantagé avec un taux d'accès de 45\% (Kengne F, 2018). Les sources d'approvisionnement en eau potable se présentent sous différentes formes notamment bornes fontaines, puits, sources, rivières et fleuves, eaux de pluies et varient en fonction des différentes zones d'habitation ( Tanawa et al., 2002). Cela a un impact direct sur la santé de la population. Selon le Global Water Partnership Central Africa (2010), plus de 10 millions de camerounais ont souffert de maladies hydriques entre 2003 et 2006 ce qui a entrainé des dépenses évaluées à 65975 FCFA/ménage. Ainsi le problème ne se pose plus en termes de disponibilité mais de gouvernance proactive, préventive et coordonnée (Graffy, 2006). Au Cameroun, la gestion de l'eau est centralisée et ne prend pas en compte le milieu rural ce qui y explique la croissance des maladies hydriques (Tantoh et Simatele, 2017). L'accès à l'eau potable est abandonné aux ONG, les organisations communautaires et les élites qui mettent sur pied des projets à viabilité irrégulière (Breuil L., 2001). Il manque une coordination efficace des 
actions de différents acteurs (makkaoui R. 2004) Comme conséquences, les maladies hydriques figurent parmi les pathologies les plus récurrentes dans les milieux ruraux (Ofouemé B., 2010), et plus particulièrement ceux des Hautes Terres de l'Ouest très densément peuplés et en connexion étroite avec les villes. L'évolution des maladies hydriques est donc calquée, non pas sur la présence ou non de l'eau potable, mais de la façon dont cette eau est gérée (Kouam G.et al, 2009). Le village Bangoua par exemple a bénéficié d'un projet de réhabilitation du réseau scanwater. Cependant, force est de constater que le nombre de personnes souffrant de maladies hydriques oscille avec la gouvernance de ce projet par les parties prenantes : la bonne gouvernance du projet est en effet corrélée à une baisse significative des maladies hydriques dans le centre de santé de la localité. Par contre, une mauvaise gouvernance entraine une augmentation. L'objectif de cet article est donc de montrer ce rapport étroit entre la gouvernance de l'eau et l'évolution des maladies en milieu rural. Après une mise en évidence du déficit d'eau à Bangoua, l'évolution des modes d'approvisionnement permet d'établir les cas de déficit de gouvernance entrainant la hausse des maladies hydriques et les cas de bonne gouvernance conduisant à leur baisse.

\section{Méthodologie}

\subsection{Zone d'étude}

Cette étude a été menée dans le village Bangoua, situé dans la région de l'Ouest Cameroun et plus précisément dans l'arrondissement de Bangangté. Il s'étale entre $5^{\circ} 9$ et $5^{\circ} 15$ de Latitude Nord et entre $10^{\circ} 24$ et $10^{\circ} 30$ de Longitude Est, pour une superficie de $84 \mathrm{~km}^{2}$ et une population d'environ 20 000 habitants. C'est l'un des sept villages de l'arrondissement de Bangangté (figure 1). 


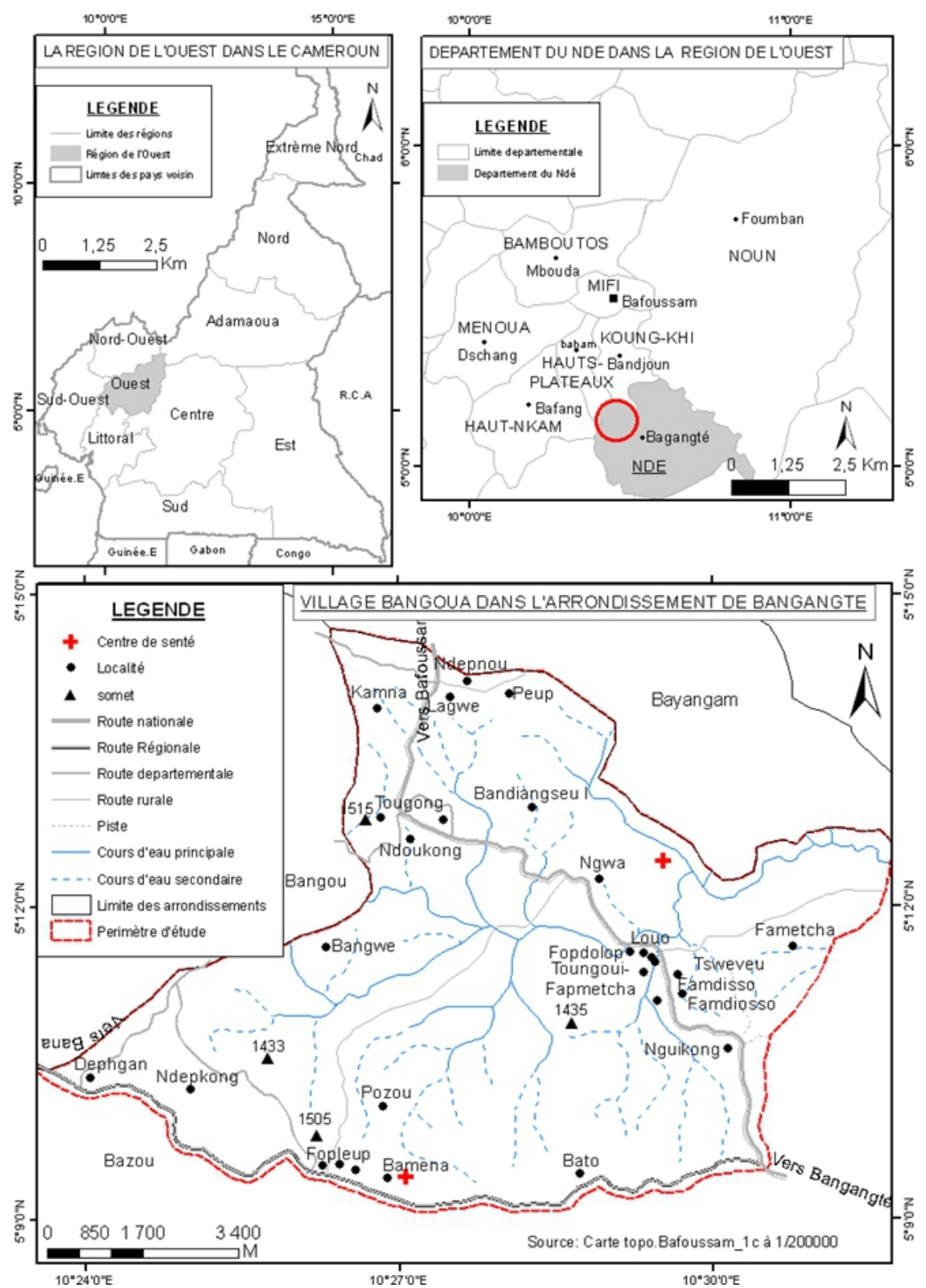

Figure 1 : Localisation de la zone d'étude

Sur le plan physique, la position privilégiée de Bangoua à mie flanc du mont Bangou (1924 m) laisse voir de nombreux micro bassins-versants perchés dont les plus importants sont celui de Kamna (1780 m) et Peup (1753 $\mathrm{m})$. Les affleurements de granite n'offrent aucune chance à la pénétration de l'eau dans le sol. Le relief fortement disséqué confère les conditions de réalisation des captages superficiels par gravité à la faveur des tranchées et des collecteurs au pied des bassin-versants. Les sols ferralitiques et indurés ne 
permettent pas au climat équatorial de type camerounéen d'altitude de saturer de manière optimale la nappe phréatique. La pluviométrie moyenne annuelle de $1450 \mathrm{~mm}^{3}$ sur neuf mois n'est pas suffisante comme en témoigne le régime irrégulier de la majorité des cours d'eau.

$\mathrm{La}$ population rurale s'active dans l'agriculture irriguée particulièrement les produits maraichers en vue d'approvisionner les métropoles Douala et Yaoundé. A cela s'ajoute les activités autour du bâtiment qui constituent autant de pression sur les ressources hydriques.

\subsection{Collecte des données}

Le registre d'abonnement au réseau d'eau réhabilité du Comité Eau du Groupement Bangoua (COMEGBA) a permis de choisir les quartiers enquêtés sur la base du nombre de ménage, le nombre d'abonné (Tougong, Toumveu, Kamna et Bandiangsseu) tandis que celui des malades du centre de santé de Bangoua a permis de dénombrer les personnes souffrant de maladies hydriques dans ces quartiers. Le nombre de ménage enquêté, choisi au hasard a été déterminé par la méthode de quota suivant la formule : nombre de ménage $\times 25 \%$ (tableau 1$)$.

Le questionnaire a permis d'identifier les mécanismes de gouvernance, déceler les problèmes rencontrés par les populations et le comité de gestion et mesurer l'impact de la présence/absence de l'eau sur la santé des populations.

Tableau 1 : Nombre de ménages enquêtés par quartier

\begin{tabular}{|c|c|c|c|c|c|}
\hline $\begin{array}{l}\text { Nom des } \\
\text { quartiers }\end{array}$ & $\begin{array}{l}\text { Date de } \\
\text { création } \\
\text { du réseau } \\
\text { d'eau }\end{array}$ & $\begin{array}{l}\text { Date de } \\
\text { réhabilitation } \\
\text { du réseau } \\
\text { d'eau }\end{array}$ & $\begin{array}{l}\text { Nombre } \\
\text { de } \\
\text { ménages }\end{array}$ & $\begin{array}{l}\text { Pourcentage } \\
\text { en } \%\end{array}$ & $\begin{array}{l}\text { Nombre de } \\
\text { ménage } \\
\text { enquêté }\end{array}$ \\
\hline Tougong & 1984 & 2006 et 2014 & 152 & 25 & 38 \\
\hline Toumveu & 1984 & 2006 et 2014 & 142 & 25 & 35 \\
\hline Kamna & 1984 & 2006 et 2014 & 158 & 25 & 39 \\
\hline Badiangsseu & 1984 & 2017 & 160 & 25 & 40 \\
\hline Totaux & & & 612 & 100 & 153 \\
\hline
\end{tabular}

Les entretiens avec les autorités intervenant dans la gouvernance de l'eau ont permis de compléter les observations et de mieux questionner la gouvernance. Le délégué départemental du Ministère de l'Eau et de Energie (MINEE) a permis de retracer l'historique de la Scanwater à Bangoua; le chef du village Bangoua a expliqué son rôle dans la gestion du réseau réhabilité tandis que le représentant de la fondation Véolia a parlé de leurs motivations et des moyens mis en œuvre pour atteindre leurs objectifs. Le représentant du président du comité de développement a exposé leurs actions en matière d'approvisionnement en eau alors que les chefs des quartiers Tougong, Kamna, Toumveu, Bandiangsseu ont montré le degré d'implication des populations de leurs quartiers dans la gestion des projets d'eau. Les données 
techniques concernant le point de captage, les différents forages, le fonctionnement des châteaux ainsi que du réseau d'eau ont été récoltées auprès du personnel technique de la COMEGBA. L'infirmier chef du centre de santé intégré a fourni les registres et a donné sa propre perception des problèmes d'eau et des maladies hydriques engendrées.

Afin d'apprécier l'efficacité du projet sur la santé des populations, un index d'efficacité a été calculé par quartier dont la formule est : (somme des facteurs + le facteur de correction)/100. En effet étant donné que le projet a pour but l'approvisionnement en eau en qualité, en quantité et à une distance et à un prix raisonnable tel que definie par 1' OMS, des valeurs sont attribuées aux differents indicateurs (présence, quantité, qualité, distance, prix). De façon litteralle, l'indice d'efficacité est le rapport entre l'actif (ce qui est réalisé) et l'objectif (ce qui est attendu). Pour la formulation, sur la base des observations, une échelle sur 20 est élaborée avec chacun des critères ayant une pondération. La somme de tout ceci donne un score sur 20. Par la suite des variables chiffrés sont attribués à chaque critère sur 20 et c'est le score obtenu sur chaque critère dans chaque zone sur 100 qui permet d'avoir la formule. Le facteur de correction ici, met en exegue de poids et l'impact. C'est pour cela que la definition d'un facteur de correction pouvant tenir compte du nombre de personne impactée par le projet est important. Ainsi, on defini $F$ (facteur de correction) en fonction de $\mathrm{N}$ (l'effectif de la population). Pour $\mathrm{N}<10, \mathrm{~F}=0 ; \mathrm{N}>10<20, \mathrm{~F}=2 ; \mathrm{N}>20, \mathrm{~F}=4$.

Les différents facteurs à savoir la distance parcourue pour s'approvisionner en eau, la quantité d'eau, le prix $\mathrm{du} \mathrm{m}^{3}$ d'eau ont été déterminés via le questionnaire et les observations. L'analyse des échantillons d'eau prélevés au niveau des points d'eau les plus fréquentés par les ménages enquetés s'est fait au Laboratoire de Physiologie et Santé Animale de la Faculté d'Agronomie et de Sciences Agricoles suivant les critéres de classification de la qualité de l'eau selon l'OMS, et a permis de déterminer la qualité des differentes sources d'eau. Cet index varie de 0 à 100 et s'apprécie comme suit : $0=$ nulle, de 1 à $19=$ très mauvaise ; de 20 à $39=$ mauvaise ; de 40 à $59=$ passable ; de 60 à $79=$ bonne et de 80 à $100=$ très bonne.

\section{Résultats}

2.1 Insuffisance quantitative de l'eau et croissance des maladies hydriques dans les quartiers étudiés

Bangoua est alimenté par 2 chateaux d'eau. Le premier a une capacité de $50 \mathrm{~m}^{3}$ et est situé à Kamna(1732m). Il est alimenté par deux forages situés à Bayangam (village voisin) qui n'ont fonctionnés que durant deux années car vandalisés par les habitants du village voisin car declarent ils leur sous sol est exploité à leur detriment pourtant ils font face au même probleme de manque 
d'eau. Ainsi, tous les quartiers (Kamna, Bandiangsseu, Ndepnou, Peup, Langwui) rattachés à ce chateau se trouvent privés d'eau. Le second chateau d'une capacité de $27 \mathrm{~m}^{3}$ situé à $1520 \mathrm{~m}$ est alimenté par un captage et dessert huit quartiers (Tougong, Toumveu, Fapdiogsso, Fapveu, Fapdolop, Tougwui, Ndoukong, Louo dont l'effectif total de la population est de 2206 habitants. En faisant le calcul (27000litres 12610), chaque habitant dispose de 10litres/jour et c'est en deça de la norme établie.

\subsection{Conditions d'approvisionnement en eau et maladies hydriques}

Les modes d'approvisionnement sont diverses et changent en fonction des saisons. Durant la saison de pluies, l'eau de pluies est la plus consommée (27\% des ménages) tandis qu'en saison sèche, c'est plutôt celle de la source non aménagée (32\% des ménages). De façon générale, un phénomène de compensation est à relever entre la saison de pluies et la saison sèche au niveau de chaque source (figure 2). Toutefois, indépendamment des saisons, la borne

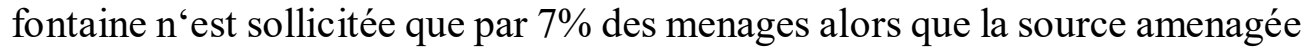
est la plus sollicitée (19\% en saison pluvieuse et $32 \%$ en saison séche). La courbe d'évolution mensuelle des maladies hydriques (figure 3) montre une augmentation du nombre de cas de maladies hydriques en saison seche et un baisse en saison de pluies.
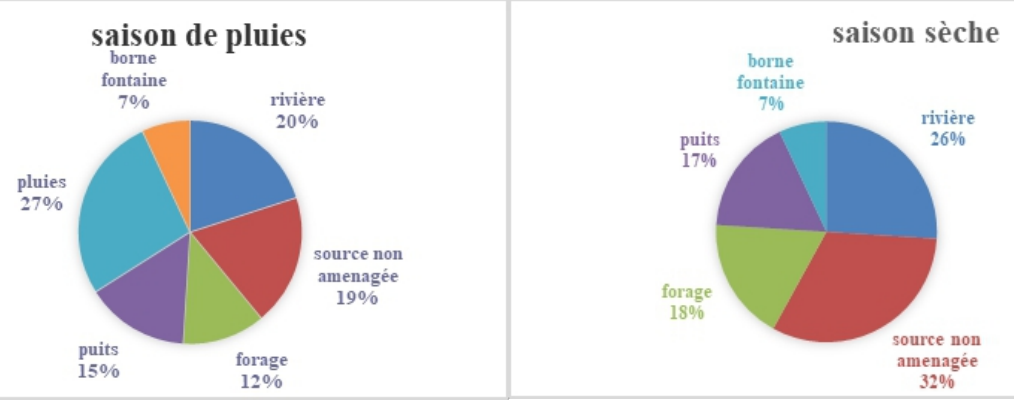

Figure 2: Répartition des ménages en fonction des modes d'approvisionnement en eau en saison de pluies (a) et en saison sèche (b)

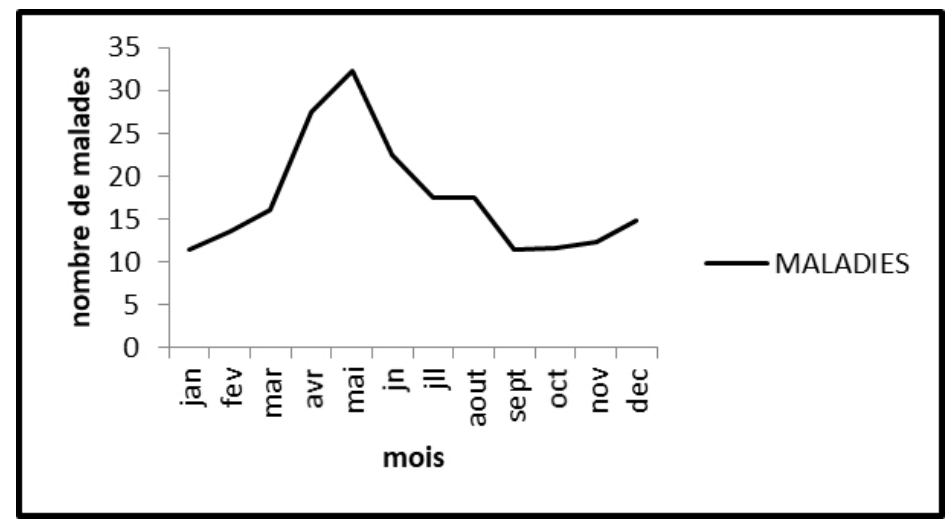

Figure 3 : Répartition mensuelle des maladies hydriques dans les quartiers enquêtés 
La fréquence d'approvisionnement varie en fonction des saisons, des sources d'eau et par quartier (tableau 2). Elle est plus élevée en saison de pluies ( 5 fois/semaine) qu'en saison sèche ( 3 fois /mois. Face à des quantités réduites dans le ménage, des stratégies sont mises en place pour économiser l'eau comme par exemple, l'utilisation des petites quantités, la réutilisation d'une même eau pour effectuer plusieurs tâches (vaisselle-lessive-nettoyage du sol-arrosage de la cour).

Dans les quartiers Tougong et Toumveu la distance moyenne (tableau 3) entre les ménages enquetés et les differentes sources d'eau est de $500 \mathrm{~m}$. À distance égale, le choix est porté sur la source non amenagée (28\% à Tougong et 25\% à Toumveu). Par contre dans les quartiers Kamna et Bandiangsseu 30\% à $5 \%$ des habitants parcourent respectivement $1,5 \mathrm{~km}$ à $2,5 \mathrm{~km}$ pour s'approvisionner en eau. Plus la distance entre le menage et le point d'eau est longue (2,5km entre la borne fontaine et le menage à Kamna et Bandiangsseu), moins le choix y est porté et plus la fréquence d'approvisionnement y est moindre ( 1 fois/mois). La repartition statistique sur les maladies hydriques obtenues au niveau du centre de santé revéle que les quartiers Kamna et Bandiangsseu enregistrent les plus grands effectifs de cas de maladies hydriques tandis que les quartiers Tougong et Toumveu enregistrent peu de cas (figure 4). A cause de la distance, les ménages choississent les points d'eau les plus proche où la fréquence d'approvisionnement sera le plus élevé. Par contre les points d'eau éloignés ne sont pas trés prisés et la fréquence d'approvisionnement est moindre et cela impacte sur l'évolution des maladies hydriques.

Tableau 2 : Fréquence d'approvisionnement en eau en fonction des saisons, des sources d'eau et par quartier

\begin{tabular}{|c|c|c|c|c|c|c|c|c|}
\hline & \multicolumn{2}{|l|}{ Tougong } & \multicolumn{2}{|l|}{ Toumveu } & \multicolumn{2}{|c|}{ Bandiangsseu } & \multicolumn{2}{|l|}{ Kamna } \\
\hline & Saison sèche & $\begin{array}{l}\text { Saison de } \\
\text { pluies }\end{array}$ & Saison sèche & $\begin{array}{ll}\begin{array}{l}\text { Saison } \\
\text { pluies }\end{array} & \text { de } \\
\end{array}$ & $\begin{array}{l}\text { Saison } \\
\text { sèche }\end{array}$ & $\begin{array}{l}\text { Saison de } \\
\text { pluies }\end{array}$ & $\begin{array}{l}\text { Saison } \\
\text { sèche }\end{array}$ & $\begin{array}{l}\text { Saison de } \\
\text { pluies }\end{array}$ \\
\hline Rivière & 2fois/semaine & 2fois/semaine & 2fois/semaine & 1fois/semaine & 3fois/mois & 2 fois/mois & 3fois/mois & 2fois/mois \\
\hline $\begin{array}{l}\text { Source } \\
\text { non } \\
\text { aménagée }\end{array}$ & 2 fois/semaine & 1 fois/semaine & 1 fois/semaine & 2fois/semaine & 2 fois/mois & 2 fois/mois & 2 fois/mois & 2 fois/mois \\
\hline Forage & 1 fois/semaine & 2 fois/semaine & 1 fois/semaine & 1fois/semaine & 1 fois/mois & 3 fois/mois & 1 fois/mois & 2 fois/mois \\
\hline Puits & 1 fois/semaine & 4fois/semaine & 1 fois/semaine & 1fois/semaine & 1 fois/mois & 3 fois/mois & 1 fois/mois & 3 fois/mois \\
\hline $\begin{array}{l}\text { Borne } \\
\text { fontaine }\end{array}$ & 1 fois/semaine & 5fois/semaine & 1 fois/semaine & 3 fois/semaine & I & I & l & I \\
\hline Pluies & l & Chaque fois & I & Chaque fois & I & $\begin{array}{l}\text { Chaque } \\
\text { fois }\end{array}$ & l & $\begin{array}{l}\text { Chaque } \\
\text { fois }\end{array}$ \\
\hline
\end{tabular}

Tableau 3 : Distance moyenne parcourue et proportion de la population en fonction des sources d'eau et des quartiers

\begin{tabular}{lllll}
\hline & Tougong & Toumveu & Bandiangsseu & Kamna \\
\hline $\begin{array}{l}\text { Rivière } \\
\begin{array}{l}\text { Source non } \\
\text { aménagée }\end{array}\end{array}$ & $500 \mathrm{~m}(25 \%)$ & $500 \mathrm{~m}(25 \%)$ & $1,5 \mathrm{~km}(23 \%)$ & $2 \mathrm{~km}(22 \%)$ \\
Forage & $500 \mathrm{~m}(28 \%)$ & $500 \mathrm{~m}(25 \%)$ & $1,5 \mathrm{~km}(30 \%)$ & $2 \mathrm{~km}(28 \%)$ \\
& $500 \mathrm{~m}(15 \%)$ & $700 \mathrm{~m}(10 \%)$ & $2 \mathrm{~km}(13 \%)$ & $2 \mathrm{~km}(15 \%)$
\end{tabular}




\begin{tabular}{lllll} 
Puits & $500 \mathrm{~m}(12 \%)$ & $500 \mathrm{~m}(20 \%)$ & $1,5 \mathrm{~km}(27 \%)$ & $1,5 \mathrm{~km}(30 \%)$ \\
Borne fontaine & $300 \mathrm{~m}(20 \%)$ & $500 \mathrm{~m}(20 \%)$ & $2,5 \mathrm{~km}(7 \%)$ & $2,5 \mathrm{~km}(5 \%)$ \\
\hline
\end{tabular}

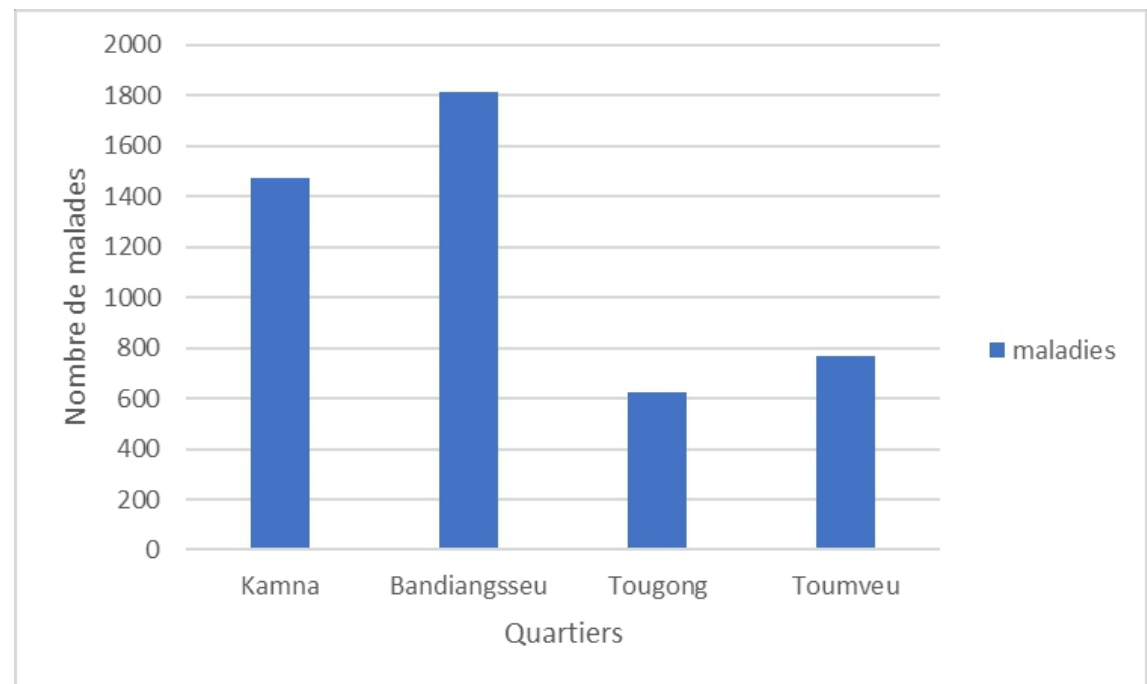

Figure 4: Repartition des maladies dans les quartiers enquetés

\subsection{Déficit de gouvernance dans la gestion de l'eau à l'échelle des ménages et presence des maladies}

Dans les ménages, l'eau est gérée suivant trois variables (tableau 4). Parmi les personnes en charge de l'eau dans les ménages, $75 \%$ sont les enfants âgés de 05 ans à 15 ans. Les adultes n'occupent que 25\%. C'est durant la saison de pluie que tous les membres de la maison ( 05 ans et + ) s'en charge car l'eau est à proximité du ménage. Les observations au point de collecte d'eau revèlent que le nettoyage des récipients se fait par une agitation mécanique, du plastique ramassé autour du point d'eau permet de fermer emertiquement le bidon. Après puisage de l'eau, un temps de jeu est observé avant le retour. Durant cette période l'eau recueillie dans les récipients ouverts est exposée à toutes formes de pollution, une couche de poussière est observée au dessus, des morceaux de feuilles tombées dans l'eau sont balayées par un coup de main introduit dans le récipient. En ce qui concerne le matériel d'approvisionnement et de stockage de l'eau, $90 \%$ de ménages préférent les bidons (5 à 20 litres) en saison sèche puisqu'ils sont faciles à transporter par les enfants. La durée de stockage est de 5jours minimum. Cependant en saison de pluie, le fû̀t est le plus sollicité ( $95 \%$ de ménages) car permet à l'aide des gouttières de recueillir une très grande quantité d'eau du ruissellement sur les toits de maisons. Durant la saison pluvieuse, ces fûts se trouvent à l'extérieur de la maison, non couverts et nettoyés uniquement au début de chaque saison de pluies. Ainsi l'eau y est stockée pour une très longue durée (toute la saison). 
Des dépots sont observés au fond de ces fûts devenus des biotopes pour les insectes.

Tableau 4 : Mode gestion de l'eau dans le ménage

\begin{tabular}{|c|c|c|c|c|}
\hline $\begin{array}{l}\text { Personne en } \\
\text { charge de l'eau }\end{array}$ & $\begin{array}{l}\text { Ordre d'utilisation } \\
\text { du matériel }\end{array}$ & $\begin{array}{l}\text { Matériel et durée de } \\
\text { stockage }\end{array}$ & $\begin{array}{l}\text { Mode } \\
\text { traitement }\end{array}$ & $\begin{array}{l}\text { Stratégie } \\
\text { d'économie }\end{array}$ \\
\hline $\begin{array}{l}\text {-Enfants : } 75 \% \\
\text {-Adultes :25\% } \\
\text {-Vieux : / } \\
\text {-Tout le monde : }\end{array}$ & $\begin{array}{l}\text {-Bidon : premier } \\
(90 \%) \\
\text {-Cuvette : deuxième } \\
(4 \%) \\
\text {-Seau : troisième (3\%) } \\
\text {-Bouteille : quatrième } \\
(3 \%) \\
\text {-fut :95\% en saison de } \\
\text { pluies }\end{array}$ & $\begin{array}{l}\text {-Bidon : premier } \\
(90 \%) ; 5 \text { jours } \\
\text {-Cuvette : deuxième } \\
(4 \%) ; 3 \text { jours } \\
\text {-Seau : troisième (3\%) } \\
\text {;1jour } \\
\text {-Bouteille : quatrième, } \\
\text { (3\%) } 2 \text { jours } \\
\text {-fut: } 95 \% \text { en saison de } \\
\text { pluies; toute la saison }\end{array}$ & $\begin{array}{l}\text {-Aucun :80\% } \\
\text {-Ebullition :20\% } \\
\text {-Javellisation:/ } \\
\text {-Filtration:/ }\end{array}$ & $\begin{array}{l}\text {-Utilisation des } \\
\text { petites quantités } \\
\text { d'eau : } 66 \% \\
\text { - Une même eau } \\
\text { pour plusieurs } \\
\text { usages : } 34 \%\end{array}$ \\
\hline
\end{tabular}

Le traitement de l'eau douteuse aurait permis de réduire les vulnérabilités. Or, seuls $20 \%$ des ménages recourt à l'ébullition tandis que $80 \%$ des ménages les ignorent en se fondant sur la conviction que leur eau est potable. Face à ce faible taux de traitement de l'eau, une analyse des differentes échantillons d'eau est nécessaire afin de determiner la qualité de l'eau consommée.

L'analyse des échantillons d'eau fait ressortir deux catégories de sources : les sources moins polluées constituées de la borne fontaine et le forage, les sources très polluées constituées du puits, de la source non aménagées et de la rivière (tableau 5).

Tableau 5: Microbes spécifiques isolés dans les échantillons d'eau au ml/unité par source

$$
\text { d'eau }
$$

\begin{tabular}{llllllll}
\hline & $\begin{array}{l}\text { Enterobacteria } \\
\text { spp }\end{array}$ & E.colli & $\begin{array}{l}\text { Streptococcus } \\
\text { spp }\end{array}$ & $\begin{array}{l}\text { Salmonella } \\
\text { spp }\end{array}$ & $\begin{array}{l}\text { Shigella } \\
\text { spp }\end{array}$ & $\begin{array}{l}\text { Staphylococcus } \\
\text { spp }\end{array}$ & Conclusions \\
\hline $\begin{array}{l}\text { Source } \\
\text { non } \\
\text { aménagée }\end{array}$ & 150 & 100 & 00 & 75 & 00 & 03 & Très polluée \\
$\begin{array}{l}\text { Puits } \\
\text { Rivière }\end{array}$ & 150 & 100 & 01 & 12 & 00 & 11 & $\begin{array}{l}\text { Très polluée } \\
\text { Très polluée }\end{array}$ \\
$\begin{array}{l}\text { Borne } \\
\text { fontaine }\end{array}$ & 05 & 100 & 40 & 50 & 20 & 06 & $\begin{array}{l}\text { Acceptable } \\
\text { avec peu de } \\
\text { risque } \\
\text { Acceptable } \\
\text { avec peu de } \\
\text { risque }\end{array}$ \\
\hline
\end{tabular}

Au regard de la qualité de l'eau des differents sources et de l'évolution des maladies dans les differents quartiers (figure 4), il y a lieu d'établir un rapport entre la qualité de l'eau des sources et les maladies hydriques. 


\section{4 Évaluation des projets d'adduction en eau potable et leur impact sur les maladies hydriques}

\subsubsection{De l'échec du projet Scanwater au projet de rehabilitation du réseau scanwater}

Le projet Scanwater était cofinancé par l'Etat danois et l'Etat camerounais. L'Etat danois avait pour tâche de financer l'installation des stations Scanwater pour l'adduction en eau potable et de former quelques techniciens pour le suivi du projet. Le suivi et l'entretien des ouvrages devraient être assuré par l'Etat Camerounais. Ce projet a permis au village Bangoua de bénéficier de 16 stations scanwater qui n'ont fonctionné que le temps des essais et de la réception à cause de plusieurs difficultés dont : l'insuffisance des études géophysiques, l'usage d'un matériel de moindre qualité, le mauvais choix de la période pour l'exécution des ouvrages, l'inadaptation des types d'ouvrage et du matériel de travail. De plus la mauvaise répartition des ouvrages, la non implication des populations, l'absence de suivi après exécution ont créé des conditions favorables aux actes de vandalisme enregistrés au niveau des ouvrages de scanwater. Toutes ces difficultés ont été à l'origine de l'échec du projet en 2003. Toutefois, elles donneront naissance plus tard à une prise de conscience qui aboutira à un projet de réhabilitation en 2005 (figure 5). La réhabilitation en 2005, par la fondation Véolia, a permis la réfection des infrastructures et l'assignation de nouveaux rôles aux parties prenantes.

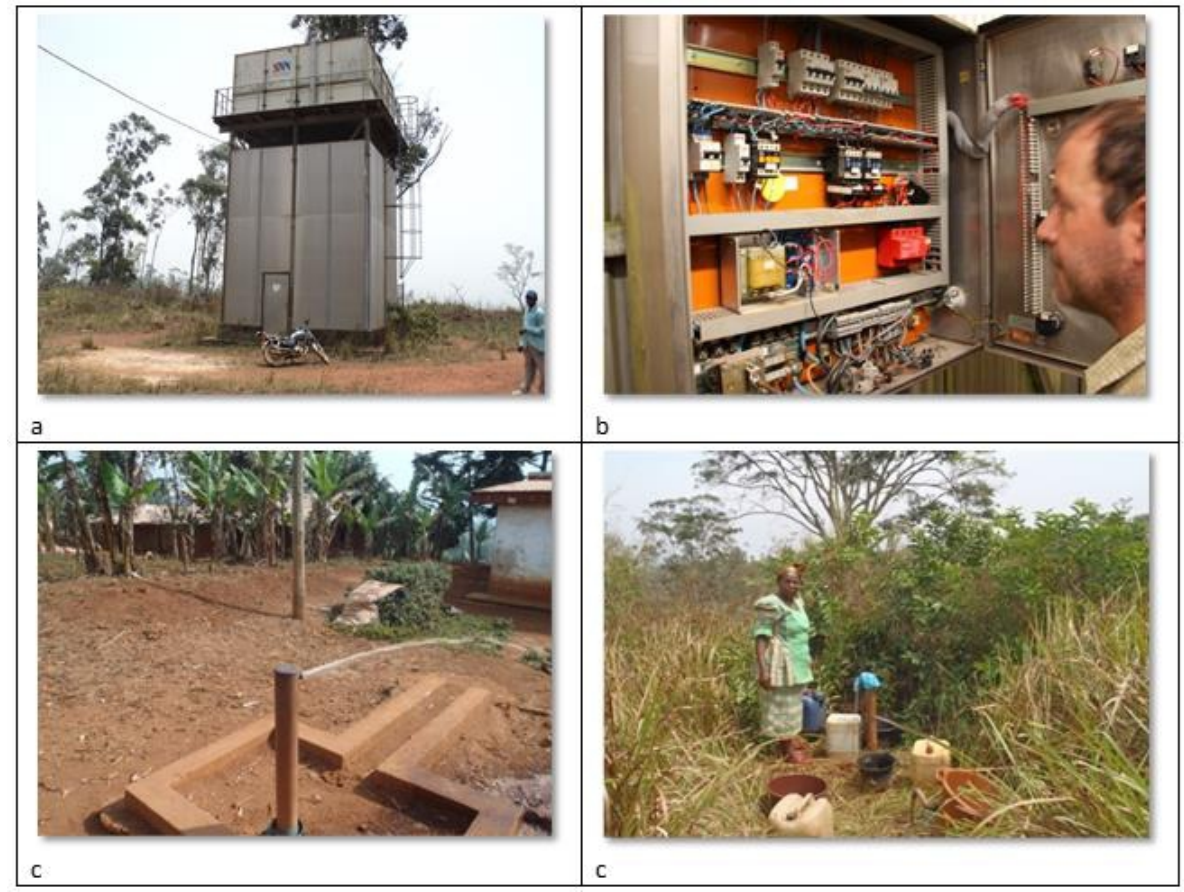

Figure 5 : Photographie de la réhabilitation du réseau scanwater

(a : château d'eau, b: tableau de commande, $\mathbf{c}$ : bornes fontaines fonctionnelles) 
Bien que rehabilité, la gestion du reseau scanwater fera face à de nombreuses insuffisances.

\subsubsection{Projet de réhabilitation du réseau d'eau Scanwater et les nouveaux déficits de gouvernance}

Un taux d'abonnement faible et augmentation des factures impayés

$\mathrm{Au}$ fil du temps, le taux d'abonnement, bien qu'il progresse, demeure tres faible(tableau 6). il passe de 17,03 en 2009 à 22,95 en 2018. De l'autre coté, le pourcentage de factures impayées croit rapidement. Il est passé de $33,76 \%$ en 2009 à $100 \%$ en 2018.

Tableau 6 : Nombre d'abonné au réseau et factures impayées

\begin{tabular}{|c|c|c|c|c|c|c|c|c|c|c|}
\hline Années & 2009 & 2010 & 2011 & 2012 & 2013 & 2014 & 2015 & 2016 & 2017 & 2018 \\
\hline Nombre de ménages & 452 & 467 & 496 & 508 & 600 & 602 & 603 & 605 & 607 & 610 \\
\hline Nombre de ménage abonné & 77 & 85 & 84 & 84 & 84 & 84 & 95 & 103 & 133 & 140 \\
\hline Taux d'abonnement (\%) & 17,03 & 18,2 & 16,93 & 16,53 & 14 & 13,95 & 15,75 & 17,02 & 21,91 & 22,95 \\
\hline Nombre de factures impayées & 26 & 47 & 83 & 84 & 84 & 84 & 95 & 103 & 133 & 140 \\
\hline $\begin{array}{l}\text { Pourcentage de factures } \\
\text { impayées en \% }\end{array}$ & 33,76 & 55,29 & 98,8 & 100 & 100 & 100 & 100 & 100 & 100 & 100 \\
\hline
\end{tabular}

A cause de ce manque de moyens financiers, les factures d'électricité ne sont pas réglées, les pannes ne sont pas réparées d'où l'arrêt du fonctionnement du comité de gestion en 2012. Le système de captage caractérisé par un fonctionnement irrégulier est désormais géré par un technicien à sa guise parce que les frais d'abonnement sont variables, le payement des frais de consommation est irrégulier et se fait sous forme de gratification du technicien. Même avec une seconde réhabilitation du réseau en 2014, la satisfaction des attentes des populations n'a pas été atteinte à cause de l'absence d'un comité de gestion qui entrainera l'arrêt du fonctionnement du réseau d'eau dans la partie Nord et poussera le technicien à perdurer dans sa gestion par gratification directe dans la partie sud.

\section{Disparité spatio-temporelle du taux d'abonnement en eau}

$\mathrm{Au}$ fur et mesure que le temps passe, le taux d'abonnement avec eau baisse tandis que celui sans eau augmente (figure 6 et 7). Globalement, le taux d'abonnement sans eau est passé de $27,5 \%$ en 2009 à $91,75 \%$ en 2018 et le taux d'abonnement avec eau est passé de $43 \%$ en 2009 à 8,25 en 2019. Tougong et Toumveu sont les seuls quartiers dans lesquels le taux d'abonné avec eau est médiocre 14\% pour Tougong et 10\% pour Toumveu). Dans les autres quartiers, il est presque nul( $1 \%$ pour Kamna; $8 \%$ pour Bandiangsseu) 


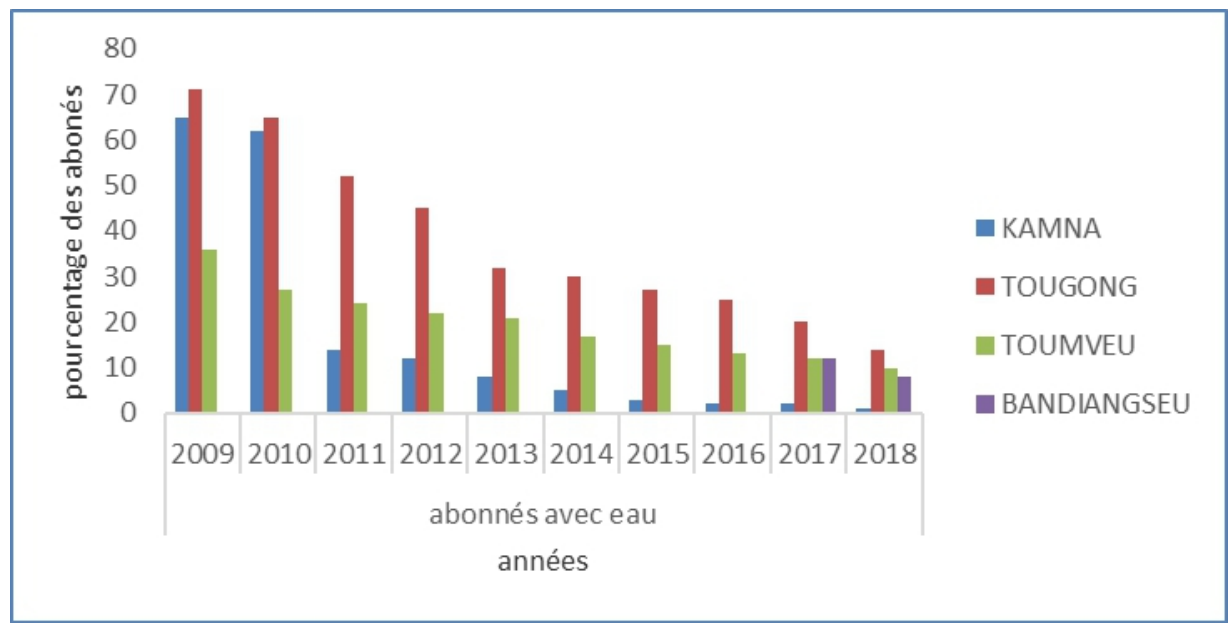

Figure 6 : Evolution du taux de ménage abonné avec eau de 2009 à 2018 à Bangoua

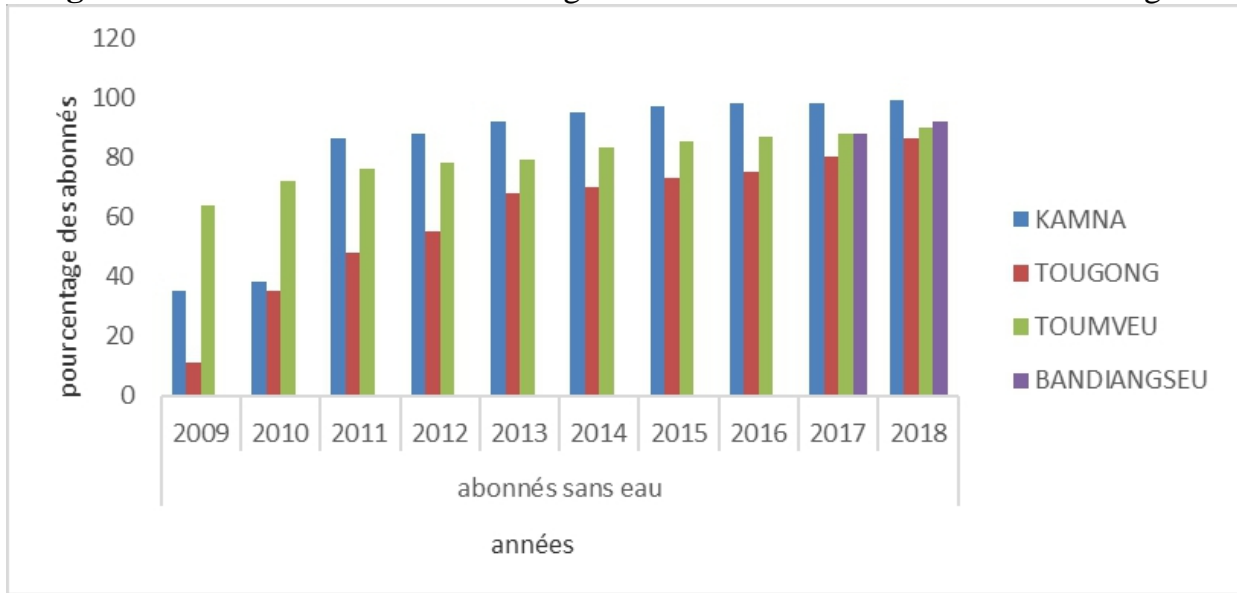

Figure 7 : Evolution du taux de ménage abonné sans eau de 2009 à 2018 à Bangoua

Cette disparité est justifiée par les pratiques de mauvaise gestion. Il existe une discrimination dans la distribution de l'eau dans les quartiers avec une préférence pour le quartier Tougong qui abrite la chefferie et Toumveu où réside le technicien du réseau. Outre les actes de vandalisme sur le réseau, les contraintes naturelles entrainent la réduction du débit, en saison sèche.

\section{Les pertes en eau: un véritable manque à gagner}

L'augmentation du nombre d'abonné sans eau s'explique aussi par les pertes en eau le long du réseau (tableau 7). Les différents rélevés montrent que les quantités d'eau entrées dans le château sont différentes de celles sorties. De même, celles facturées ne correspondent pas aux quantités sorties du château. Les pertes sont donc énormes, elles s'évaluent à $1738 \mathrm{~m}^{3}$ pour le château de Kamna or la quantité d'eau facturée n'est que de $220 \mathrm{~m}^{3}$. Ceci cause d'un véritable manque à gagner évalué à 521400 fcfa hormis les frais 
d'abonnement et de location compteur pour le mois de janvier et de février 2009. Les observations faites au niveau des chateaux relevent que les equipements du reseau sont vieux, mal entretenu. Au niveau du reseau, des connexion illicites sont notées, les tuyaux sont cassés.

Tableau 7 : Récapitulatif des entrées, sorties et facturation des volumes d'eau des principaux châteaux

\begin{tabular}{lllllll}
\hline Châteaux & $\begin{array}{l}\text { Mois en } \\
\mathbf{m}^{\mathbf{3}}\end{array}$ & $\begin{array}{l}\text { Entrées } \\
\mathbf{e n ~}^{\mathbf{3}}\end{array}$ & $\begin{array}{l}\text { Sorties } \\
\mathbf{e n ~}^{\mathbf{3}}\end{array}$ & $\begin{array}{l}\text { Facturation } \\
\mathbf{e n ~}^{\mathbf{3}}\end{array}$ & $\begin{array}{l}\text { Pertes totales } \\
\mathbf{e n ~}^{\mathbf{3}}\end{array}$ & $\begin{array}{l}\text { Evaluation des } \\
\text { pertes en fcfa }\end{array}$ \\
\hline Kamna & Janvier & 1003 & 1000 & 135 & 868 & 260400 \\
& Février & 955 & 887 & 85 & 870 & 261000 \\
\multirow{4}{*}{ Bangoua } & Totaux & $\mathbf{1 9 5 8}$ & $\mathbf{1 8 8 7}$ & $\mathbf{2 2 0}$ & $\mathbf{1 7 3 8}$ & $\mathbf{5 2 1} \mathbf{4 0 0}$ \\
& Janvier & 1097 & 605 & 489 & 608 & 182400 \\
& Février & 1132 & 1075 & 404 & 728 & 218400 \\
& Totaux & $\mathbf{2 1 7 2}$ & $\mathbf{1 7 3 7}$ & $\mathbf{8 9 3}$ & $\mathbf{1 3 3 6}$ & $\mathbf{4 0 0} \mathbf{8 0 0}$ \\
\hline
\end{tabular}

\section{Le deficit de transparence}

Le déficit de transparence est aussi mentionné au niveau de l'élection des membres du comité de gestion. En effet, ils ne sont pas élus au bulletin secret par l'assemblée générale. Le chef du village copte plus de $50 \%$ des membres. Cela crée une frustration chez les populations qui ne peuvent contester la décision du chef. Au lieu d'écouter et de prendre en compte les plaintes des populations, les membres du comité de gestion procèdent plutôt par intimidation.

Le manque de moyens financiers a entrainé l'arrêt du fonctionnement du comité de gestion laissant place depuis 2012 à un technicien qui assure l'entretien du réseau d'eau sans rendre compte. Le réseau de Bangoua, à nouveau réhabilité en 2015 et celui de Bandiangsseu réhabilité en 2017 sont gérés par le même technicien en raison de l'absence d'un comité de gestion. Bien que le nombre d'abonnés connaisse une augmentation, aucune facture n'est payée. Les abonnements et les frais de consommation sont payés à volonté et directement au technicien.

\section{Une participation insuffisante de la population}

A cela s'ajoute une participation insuffisante de la population car elle éprouve des difficultés à s'approprier le projet, le manque de moyens financiers, l'irresponsabilité de certains membres du comité de gestion, l'âpreté au gain des techniciens qui par manque de motivation, gère le réseau d'eau à leur guise et sur la base de la corruption. Et enfin l'absence du principe d'inclusion de toutes les couches sociales. Tous les villageois ne sont pas consultés lors de l'implantation des points d'eau ainsi que dans leur gestion.

Ces projets d'eau avaient pour but de fournir de l'eau potable aux populations et de ce fait, éviter que ces dernières ne fassent recours aux sources non potables. De la gestion du projet Scanwater à celui du projet de 
réhabilitation du réseau scanwater, plusieurs défaillances ont empêché l'atteinte de cet objectif et l'évolution des maladies hydriques en témoigne. Le calcul de l'index d'efficacité permet d'établir cette relation.

\subsection{Relation entre la gouvernance et l'évolution des maladies hydriques}

Sur 6789 malades enregistrés entre 2002 à 2018, 4675 souffraient de maladies hydriques dont 1127 cas gastro-entérite, 1477 cas de typhoïde, 2071 cas de dysenterie amibienne. La répartition statistique montre que la maladie la plus récurrente est la dysenterie amibienne avec une proportion de $44 \%$ (figure 8).

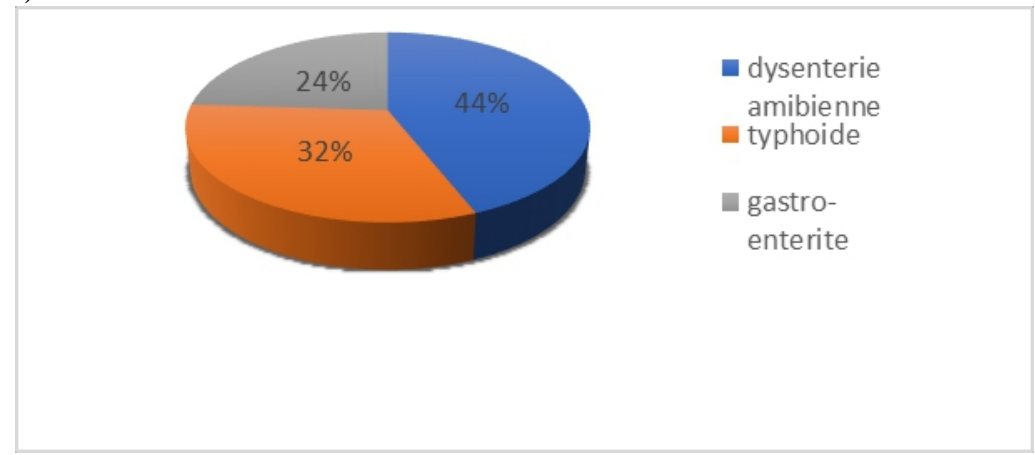

Figure 8 : Répartition des maladies hydriques

L'index d'efficacité de la gouvernance de l'eau a été calculé pendant les périodes :

- Avant le projet de réhabilitation (2003-2005) ;

- Pendant le premier projet de réhabilitation (2006-2008);

- Après le premier projet de réhabilitation (2009-2013);

- Durant la deuxième réhabilitation (2014-2015) ;

- Après le deuxième projet de réhabilitation (2016-2018) (tableau 8.).

Cet index a été croisé avec l'évolution des maladies hydriques (figure 9) et le résultat permet d'établir la relation entre la gouvernance de l'eau et l'évolution des maladies hydriques. Lorsque la courbe de l'index d'efficacité croit, celle des maladies décroît. C'est observé lors des périodes de bonne gestion du projet, c'est-à-dire où la fourniture de l'eau est régulière, les factures payées, les pannes de réseau réduit ou immédiatement pris en charge par des techniciens actifs. Après la mise en œuvre effective du projet, la courbe des maladies tend à croitre tandis que celle de l'index d'efficacité décroit. C'est le temps des ruptures de fournitures d'eau, le temps des pannes, de vandalisme et des factures impayées. Le nombre d'abonné sans eau est plus élevé et la gestion du réseau assurée par des techniciens de circonstance moins engagés. 
De façon général, la gouvernance de l'eau à un impact sur l'évolution des maladies dans les différents quartiers. Dans les quartiers Tougong, Toumveu et Kamna, bénéficiaires du premier et deuxième projet de réhabilitation, les périodes avant, pendant et après le projet, notamment le premier, sont bien matérialisées. Le quartier Bandiangsseu n'a bénéficié d'une réhabilitation du réseau qu'en 2017, ce qui explique la baisse du nombre de cas de maladies hydriques à partir de cette année.

Tableau 8: Index* d'efficacité moyen par période de gouvernance par quartier

\begin{tabular}{llllll}
\hline $\begin{array}{l}\text { Période de } \\
\text { gouvernance }\end{array}$ & la & Quartiers & & & \\
\cline { 2 - 5 } & Tougong & Toumveu & Kamna & Bandiangsseu \\
\hline $\mathbf{2 0 0 3 - 2 0 0 5}$ & 0 & 0 & 0 & 0 \\
$\mathbf{2 0 0 6 - 2 0 0 8}$ & 42 & 41 & 40 & 0 \\
$\mathbf{2 0 0 9 - 2 0 1 3}$ & 38 & 34 & 30 & 0 \\
$\mathbf{2 0 1 4 - 2 0 1 6}$ & 20 & 21 & 16 & 15 \\
$\mathbf{2 0 1 7 - 2 0 1 8}$ & 14 & 20 & 3 & 18 \\
\hline
\end{tabular}

*Cet index varie de 0 à 100 et s'apprécie comme suit : $0=$ nulle, de 1à $19=$ très mauvaise ; de 20 à $39=$ mauvaise ; de 40 à $59=$ passable ; de 60 à $79=$ bonne et de 80 à $100=$ très bonne.

L'index d'efficacité moyen par quartier suivant les différentes périodes (tableau 8) montre que la relation entre la gouvernance de l'eau et les maladies hydriques est significative. Cette corrélation varie de passable à mauvaise suivant les périodes car lorsque la gouvernance est bonne, les maladies hydriques régressent ; par contre elles augmentent lorsque la gouvernance est mauvaise. 


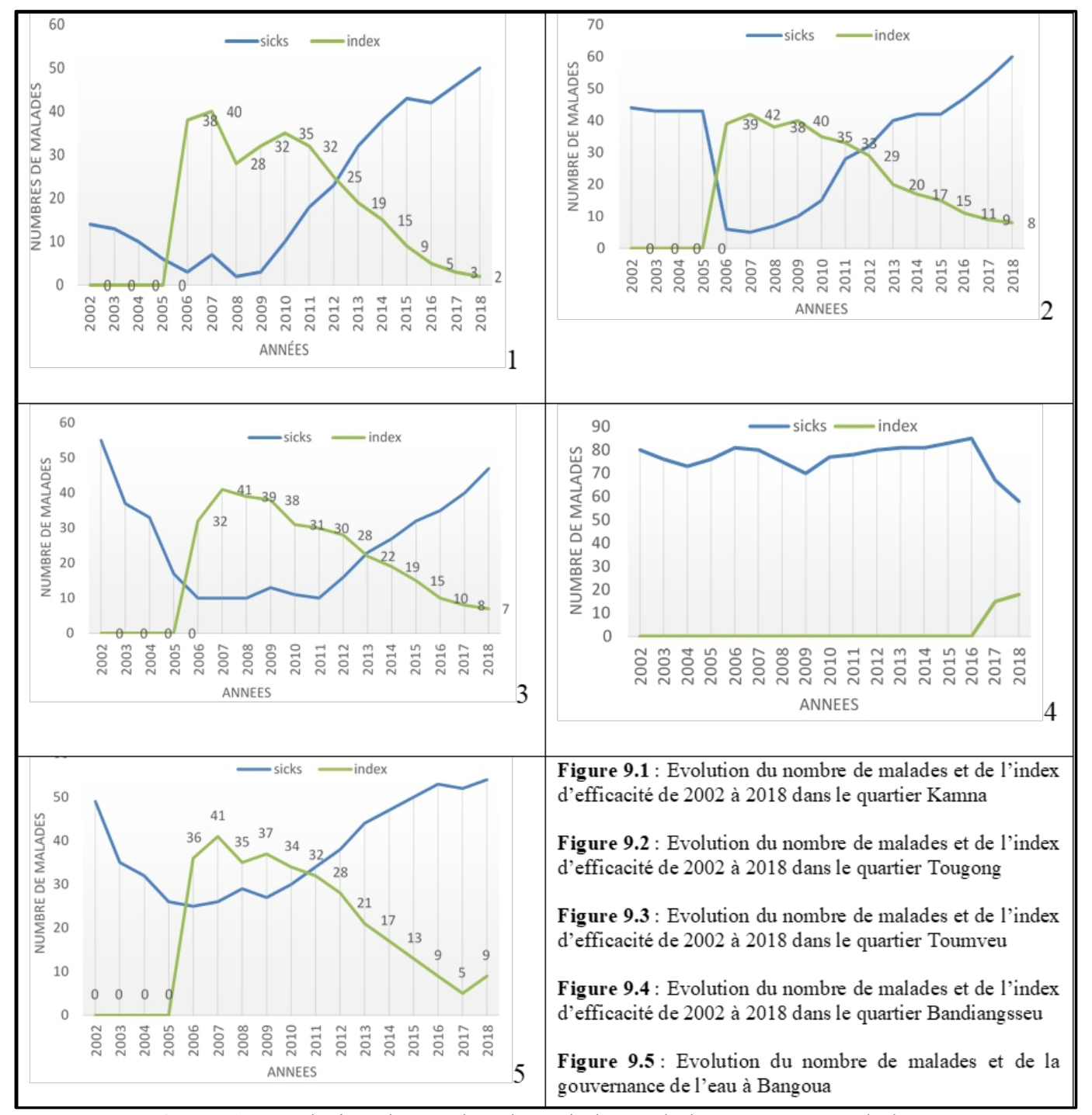

Figure 9 : Evolution du nombre de malades et de la gouvernance de l'eau

\section{Discussion}

Il ressort de cette étude que les conditions d'accés et d'approvisionnement en eau sont plus faciles en saison pluvieuse qu'en saison séche. Car en saison pluvieuse, l'eau est omniprésente, l'approvisionnement se fait à chaque fois pourtant en saison séche il faut aller la chercher et c'est pénible. L'augmentation des maladies hydriques en saison séche et sa baisse en saison pluvieuse peut être une suite logique de cette situation.

Parmis les sources d'approvisionnement, la source non aménagée est la preferée car elle a un bon goût, de génération en génération, les habitants la consomment, elle est gratuite. En milieu rural, la source non aménagée est un lieu sacré où se trouvent les dieux, l'environnement y est saint, il est interdit 
d'y mener toutes activités (lessive, vaisselle, bain) pouvant occasionner la pollution de l'eau. De même, dans les perceptions locales, l'eau des sources remontant du sol par capillarité est plus purifiée que toute autre. Or de l'analyse des echantillons d'eau, il ressort que la source non aménageé figure parmis les sources d'eau polluées d'où la croissance des maladies hydriques.

La proximité par rapport à un point d'eau contribue à l'augmentation des quantités d'eau car la fréquence d'approvisionnement est grande. Cela peut expliquer le fait que les ménages proches des points $d^{\prime}$ eau souffrent moins de maladies hydriques que ceux éloignés. Lorsque la distance entre le ménage et le point d'eau est grande, le risque de contamination au cour du transport est élevé, la fréquence d'approvisionnement est reduite, la durée de stockage augmente. Tout ceci accroit le rythme de multiplication des germes pouvant nuire à la santé des populations. L'irrégularité d'approvisionnement et la distance à parcourir impactent sur les quantités et les qualités d'eau consommées par personne par jour et accroit le risque de survenu des maladies hydriques. Les résultats des travaux de Daffé et Diagne (2009) au Sénégal montrent plutôt l'impact de la pauvreté sur les quantités et la qualité de l'eau. Pour Ofouémé (2010), la pénibilité s'accroît avec la marginalisation de l'eau dans les programmes de développement, le manque de concrétisation des actions envisagées pour améliorer les conditions de vie des populations.

Le deficit de gouvernance dans la gestion de l'eau à l'echelle des ménages présente des risques de contamination de l'eau car la probabilié que les mesures d'hygiène ne soient pas respectées est grande vue la jeunesse des principaux acteurs en charge de l'approvisionnement en eau des ménages; l'absence de mesures d'hygiène au niveau des bidons, matériel d'approvisionnement et de stockage d'eau de prédilection durant la saison séche materialisée par la présence des pointillées noirs et d'une couche marronne à l'interieur du recipient. En saison pluvieuse, le matériel de stockage est le fût. Il est exposé à toutes formes de pollution car situé hors de la maison, non couvert. La longue durée de stockage, l'absence de nettoyage font du fût un milieu infecte pour la conservation de l'eau.Selon les instances onusiennes (2000), un accès à l'eau potable est defini par la disponibilité d'au moins 20 litres/personnes/jour à un point d'eau situé à $300 \mathrm{~m}$ du lieu de résidence. Or dans les quartiers enquetés, les habitants doivent parcourir minimum $500 \mathrm{~m}$ pour disposer de 10 litres/personnes/jour. Face à une quantité reduite de l'eau, les mesures d'économies developpées à savoir l'utilisation des petites quantités, la réutilisation d'une même eau constitue une limite pour la bonne pratique des régles d'hygiène. Les travaux de Sackou (2012) montrent que ces differentes situations (état du matériel, conditions de transport et de stockage, absence de traitement, économie d'eau) augmentent les risques de contamination de l'eau avant son utilisation. Les resultats des travaux de Requillart (1985), Ngnikam (2007), ont mentionné que le puisage, 
le transport et le stockage de l'eau dans les ménages sont les sources de dégradation de la qualité de l'eau. Hounsounou et al. (2015) ont démontré que les diverses manipulations de l'eau constituent autant de potentiels facteurs de sa contamination et de croissance des maladies hydriques. Face à cela Djuissi et al. (2019) précisent que la mise en pratique des méthodes de traitement de l'eau pour les besoins élémentaires est une urgence. Elles pourraient permettre aux populations d'avoir de l'eau potable, mais cet objectif n'est pas toujours atteint par les projets hydrauliques.

Les projets dans le secteur de l'eau échouent parceque la population bénéficiaire est mise à l'écart dans les phases de conception, réalisation du projet pourtant essentielles pour sa pérénité car permettent de poser des actes qui repondent aux attentes des populations. En s'inscrivant dans la même logique Wade (1988), Ostrom (1990), Baland et Platteau (1996), Temgoua et al (2019) déclarent que la démarche participative permet de mettre en place une bonne gouvernance des ressources. Ainsi, il faut mettre l'accent sur le capital social au sein de la communauté car il permet de renforcer les démarches participatives et de limiter les risques de comportements opportunistes. Les résultats des travaux de Hounmenou (2006) et de Kam (2011) en Côte d'ivoire, sont conformes aux nôtres puisqu'ils révèlent que la non-participation des populations à l'installation des infrastructures hydrauliques et la non prise en compte des réalités socioéconomiques et culturelles des communautés rurales bénéficiaires expliquent l'échec des projets d'approvisionnement en eau en milieu rural.

Le faible taux d'abonnement et le non paiement des factures s'expliquent par le faible pouvoir d'achat des ménages incapables de debourser $60000 \mathrm{fcfa}$ pour l'abonnement, $300 \mathrm{fcfa}$ par $\mathrm{m}^{3}$ d'eau et $800 \mathrm{fcfa}$ pour la location compteur. Cela s'explique aussi par le refus justifiant que l'eau doit être gratuite, la confusion entre les frais d'abonnement et les frais de consommation. Ce faible taux d'abonnement est lié d'une part aux difficultés naturelles qui privent certains quartiers de bénéficier du réseau d'eau et exigent l'apport de forte somme d'argent et une logistique énorme pour la résolution du problème d'eau d'où la disparité socio spatiale du taux d'abonnement.

Les pertes en eau s'expliquent aussi par la vétusté des équipements du réseau, le mauvais entretien des infrastructures hydrauliques, l'incivisme de certains habitants qui font des connexions illicites sur le réseau ou cassent les tuyaux pour avoir de l'eau. Ces actes peuvent être évités si la bonne gouvernavce est pratiquée. Les travaux de Baron et Bonnassieux (2011) ont montré que les tensions qui se manifestent autour des points d'eau ne sont pas seulement liées à la diversité des écosystèmes. Elles sont fonction de la disponibilité des infrastructures hydrauliques, de leur état de fonctionnement et de la nature de leur mode de gestion. 
Les maladies hydriques occupent la premiere place parmis les pathologies dont souffrent les habitants des quartiers enquetés à cause de la mauvaise gouvernance de l'eau. En effet, avec la non application des critéres de la gouvernance de l'eau à savoir la participation, la transparence, la responsabilité et l'information, les menages n'ont pas acces à l'eau potable alors, les maladies hydriques augmentent. Les travaux de Kouam et al. (2006) ont montre que le probleme de bonne gouvernance (participation, transparence) empeche la gestion efficiente de la ressource eau. Les résultats de Bernard (2010) précisent que la gouvernance seule peut garantir la pérennité du couple eau-développement durable en impliquant gouvernement et citoyens. Bonnassieux (2011) quant à lui accuse plutôt le faible pouvoir d'achat des populations comme étant à l'origine de la recrudescence des maladies hydriques, car ne permet pas aux populations d'avoir accès aux sources d'eau potables. Mohamadou (2014) va au-delà des difficultés financières pour souligner l'incompétence technique et managériale comme étant la cause de l'échec des projets.

\section{Conclusion}

Cette étude a examiné les facteurs du mal fonctionnement à l'origine de l'échec des projets Scanwater et de son projet réhabilitation dans le village Bangoua des Hautes Terres de l'Ouest. Elle a mis ces facteurs en relation avec l'évolution des maladies hydriques en calculant un index d'efficacité de gouvernance de l'eau et en le croisant avec la courbe des maladies. Sur la base de l'hypothèse selon laquelle, l'évolution des maladies hydriques est calquée sur le dysfonctionnement dans le temps et dans l'espace des systèmes de gouvernance de l'eau mis en place, il a été prouvé que les caractéristiques du milieu physique contribuent à la diminution des quantités d'eau et favorisent de ce fait la croissance des maladies hydriques. Les perceptions locales poussent les populations à consommer l'eau des sources non aménagées en toute confiance. L'irrégularité d'approvisionnement et la distance à parcourir impactent sur les quantités et la qualité de l'eau consommée et accroit le risque de survenu des maladies hydriques. Cependant, il a été démontré au-delà de ces critères que le déficit de transparence ne permet pas le bon fonctionnement du projet. Aussi, l'absence de participation de la population bénéficiaire à tous les niveaux de la réalisation du projet handicape son appropriation. Tous ces facteurs de la mauvaise gouvernance des projets hydrauliques concourent à leurs échecs et à une reconquête des sources non potables occasionnant ainsi la croissance des maladies hydriques. L'index d'efficacité de la gouvernance de l'eau sur les maladies hydriques variant de 41 en 2007 à 9 en 2018 démontre à suffisance cette corrélation. Ainsi donc, il est capital de trouver des stratégies pour assurer un accès durable à l'eau aux populations vivant dans les milieux ruraux des Hautes Terres. 


\section{References:}

1. Ahmadou, K B, Amadou, D \& Richard, M. (2007). Approvisionnement en eau des ménages de Conakry. Afrique contemporaine, $221: 225-245$

2. Baechler, L. (2012). La bonne gestion de l'eau : un enjeu majeur du développement durable. L'Europe en Formation, 365, 3-21. doi:10.3917/eufor.365.0003.

3. Baron, C. \& Bonnassieu A., (2011). Les enjeux de l'accès à l'eau en Afrique de l'Ouest : diversité des modes de gouvernance et conflits d'usages. Mondes en développement, 156(4), 17-32. doi:10.3917/med.156.0017.

4. Bazié, J.B (2014). Accès à l'eau : l'Afrique entre abondance et pénurie. Après-demain, 31-32, 28-29

5. Charreton, M.B., Makkaoui, R, Olivier, P. \& Desjardins, M.R. (2006). La gouvernance des ressources en eau dans les pays en développement : enjeux nationaux et globaux. Mondes en développement, $\mathrm{n}^{\mathrm{o}}$ 135(3), 39-62. doi:10.3917/med.135.0039.

6. Daffe G. \& Diagne A, (2009). Le Sénégal face aux défis de la pauvreté ; les oubliés de la croissance, Paris, Karthala.

7. David, B. (2008). Impact des changements climatiques sur la capacité des nappes et des captages d'eau souterraine Techniques Sciences Méthodes, 3:62-75

8. Djuissi, T.D, Vogue, N., Ngwayu, C., Ebode, M. \& Nambile, S. (2019). Accès à l'eau potable et à l'assainissement : cas de la commune d'arrondissement de Douala V (Cameroun), Pan African Medical Journal. 8p

9. Enzo F. Maura M., Luciana S. (2012). Impact of climate change on waterborne diseases, 473 Ann IST SUPER SANITA 48(4): 473-487

10. Goita, A. (2014). Les bactéries pathogènes d'origine hydriques de l'épidémiologie à la prévention. Thèse de Doctorat en pharmacie, Université Mohamed V-Souissi, Rabat, Maroc, 134P

11. Guesnier, B. (2010). L'eau et le développement durable : un couple en rupture sans gouvernance sociétale et coopération décentralisée. Développement durable et territoires, 1: 1-17, DOI : https://doi.org/10.4000/developpementdurable.8376

12. Hofstra, N. (2011). Quantifying the impact of climate change on enteric water borne pathogen concentration in surface water. Current Opinion in Environmental Sustainability 3: 471-479

13. Hounmenou, B. G. (2006). Gouvernance de l'eau potable et dynamique locales en zone rurale au Bénin. Développement durable et territoires. Dossier 6, 1-x 
14. Hounsounou, E., Agassounon, M., Ayi-Fanou, L. \& Agbossou, E., (2015). Chaîne de l'eau du réseau public dans quelques quartiers précaires du sixième arrondissement de Cotonou-Bénin. La Revue Electronique en Sciences de l'Environnement 17 (3), DOI : https://doi.org/10.4000/vertigo.18824

15. Kam, O. (1998). Problématique de la gestion des infrastructures d'hydrauliques dans les projets d'approvisionnement du milieu rural en eau potable, Doctorat en Sociologie, Institut d'Ethnosociologie, Université de Cocody-Abidjan, Côte d'Ivoire.

16. Katte, V.Y., Fonteh, M.F., Guemuh, G.N. (2003). Domestic water quality in urban centers in Cameroon: a case study of Dschang in the West Province. African Water Journal, 43-54.

17. Khadga, B.S, Tatsuru, $\mathrm{K}^{2}$, Sadhana S, Yoko A, Arun, P B, Niranjan, B, Bhesh, RT , Futaba, K \& Junko S (2019). Socioeconomic Impacts of LCD-Treated Drinking Water Distribution in an Urban Community of the Kathmandu Valley, Nepal. Water, 11, 1323-1341

18. Kouam Kenmogne Guy-Romain , Hernanie G. Mpakam, Samuel Ayonghe Ndonwy, Serges L. Djomoudou Bopda et Georges E. Ekodeck, (2009). Gestion intégrée des ressources en eau et objectifs du millénaire pour le développement en Afrique : Cas du Cameroun », VertigO - la revue électronique en sciences de l'environnement,7(2).DOI : https://doi.org/10.4000/vertigo.2319

19. Mailhot, A. \& Collab (2009). Changements climatiques au Quebec méridional- approvisionnement en eau potable et santé publique : projections climatiques en matieres de précipitations et d'écoulements pour le Sud du Quebec-Resumé. Institut national de santé publique du Quebec, canada, 16p

20. Makkaoui R. (2004) gestion des ressources hydrauliques : roles et responsabilités de l'Etat et des institutions locales, les cahiers de l'Association Tiers Monde, $\mathrm{n}^{\circ} 19$

21. Mamadou I., Malam M.A., Moussa A.I., Bahari M.I, Idi M., Issaley N.A, Abba B., Abdourhamane A.T, Illo S., Mato M.W., Bouzou M et Descroix L. (2016). Difficultés d'accès à l'eau potable dans la ville de Zinder, Niger : causes, conséquences et perspectives, Afrique sciences, p 99-112

22. Ngefor, G. S. (2015). Changements institutionnels, stratégies d'approvisionnement et de gouvernance de l'eau sur les hautes terres de l'Ouest Cameroun : exemples des petites villes de Kumbo, Bafou et Bali. Thèse de Doctorat/PhD, Université Toulouse le Mirail - Toulouse II, Toulouse, France. 480P 
23. Nkengfack, H., Domguia, N. E. \& Kamajou F. (2017). Analyse des déterminants de l'offre de l'eau potable au Cameroun. 2017. hal0151011, https://hal.archives-ouvertes.fr/hal-01510111

24. Requillart, J.C., (1985). Projet d'eau potable, Rapport final, 54p

25. Sonny K., Djeri B., Anani K., Eklou-lawson M., Adjrah Y., Karou D., ameyapoh C., Souza, (2015). Evaluation de la qualité bactériologique des eaux de puits et de forage à Lomé, Togo; Journal of Applied Biosciences 91 : 8464-8469.

26. Tanawa E, Djeuda Tchapnga HB, Ngnikam E, Temgoua E, Siakeu J. 2002. Habitat and protection of water resources in suburban areas in african cities. Building and Environment, 37(3): 269-275.

27. Temgoua E., Meli V., Mekui M et Ndongson B. 2019, Rôle des Collectivités Territoriales Décentralisées dans la pérennisation des services d'eau et assainissement dans les zones non concédées : cas de la Commune de Dschang, International Journal of Biological and Chemical Sciences, 13(5): 122-132. DOI: https://dx.doi.org/10.4314/ijbcs.v13i5.10S

28. Vissin, E. W., Aimade, H. S. S., Dougnon, L. D., Sohounou, M., Atiye, E. Y., \& Atchade, G. A. A. (2017). Qualité de l'eau et maladies hydriques dans la commune de Toffo (Bénin, Afrique de l'ouest). Journal of Applied Biosciences, 106(1), 10300-10308.

29. Yolande Ofouémé-Berton, «L'approvisionnement en eau des populations rurales au Congo-Brazzaville », Les Cahiers d'Outre-Mer, $249 \mid$ 2010, 7-30. 